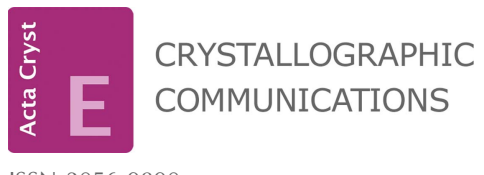

ISSN 2056-9890

Received 24 February 2021

Accepted 15 March 2021

Edited by A. Briceno, Venezuelan Institute of Scientific Research, Venezuela

Keywords: crystal structure; copper(II); tetramethylethylenediamine; 2-nitrobenzoate; Hirshfeld surface.

CCDC reference: 1910225

Supporting information: this article has supporting information at journals.iucr.org/e

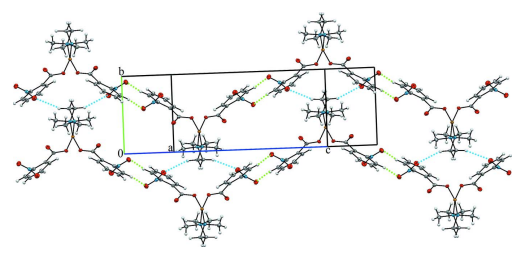

\section{Crystal structure and Hirshfeld surface analysis of a copper(II) complex containing 2-nitrobenzoate and tetramethylethylenediamine ligands}

\author{
Sevgi Kansiz, ${ }^{a *}$ Adnan M. Qadir, ${ }^{b}$ Necmi Dege, ${ }^{c}$ Li Yongxin ${ }^{d}$ and Eiad Saif ${ }^{e, f_{*}}$ \\ ${ }^{\mathbf{a}}$ Department of Fundamental Sciences, Faculty of Engineering, Samsun University, 55420, Samsun, Turkey, ${ }^{\mathbf{b}}$ Department \\ of Chemistry, College of Science, Salahaddin University, Erbil, 44001, Iraq, ' Department of Physics, Faculty of Arts and \\ Sciences, Ondokuz Mayıs University, 55139, Samsun, Turkey, dDivision of Chemistry and Biological Chemistry, \\ Nanyang Technological University, 637371, Singapore, ${ }^{\mathbf{e}}$ Department of Computer and Electronic Engineering \\ Technology, Sana'a Community College, Sana'a, Yemen, and 'Department of Electrical and Electronic Engineering, \\ Faculty of Engineering, Ondokuz Mayıs University, 55139, Samsun, Turkey. *Correspondence e-mail: \\ sevgi.kansiz@samsun.edu.tr, eiad.saif@scc.edu.ye
}

The reaction of copper(II) sulfatepentahydrate with 2-nitrobenzoic acid and $N, N, N^{\prime}, N^{\prime}$-tetramethylethylenediamine (TMEDA) in basic solution produces the complex bis(2-nitrobenzoato- $\kappa O)\left(N, N, N^{\prime}, N^{\prime}\right.$-tetramethylethylenediamine$\left.\kappa^{2} N, N^{\prime}\right)$ copper(II), $\left[\mathrm{Cu}\left(\mathrm{C}_{7} \mathrm{H}_{4} \mathrm{NO}_{4}\right)_{2}\left(\mathrm{C}_{6} \mathrm{H}_{16} \mathrm{~N}_{2}\right)\right] \quad$ or $\quad\left[\mathrm{Cu}(2 \text {-nitrobenzoate })_{2^{-}}\right.$ (tmeda)]. Each carboxylate group of the 2-nitrobenzoate ligand is coordinated by $\mathrm{Cu}^{\mathrm{II}}$ atom in a monodentate fashion and two TMEDA ligand nitrogen atoms are coordinate by the metal center, giving rise to a distorted square-planar coordination environment. In the crystal, metal complexes are linked by centrosymmetric C $-\mathrm{H} \cdots \mathrm{O}$ hydrogen bonds, forming ribbons via a $R_{2}^{2}(10)$ ring motif. These ribbons are linked by further $\mathrm{C}-\mathrm{H} \cdots \mathrm{O}$ hydrogen bonds, leading to two-dimensional hydrogen-bonded arrays parallel to the $b c$ plane. Weak $\pi-\pi$ stacking interactions provide additional stabilization of the crystal structure. Hirshfeld surface analysis, $d_{\text {norm }}$ and two-dimensional fingerprint plots were examined to verify the contributions of the different intermolecular contacts within the supramolecular structure. The major interactions of the complex are $\mathrm{O} \cdots \mathrm{H} / \mathrm{H} \cdots \mathrm{O}(44.9 \%), \mathrm{H} \cdots \mathrm{H}(34 \%)$ and $\mathrm{C} \cdots \mathrm{H}(14.5 \%)$.

\section{Chemical context}

Copper(II) carboxylate complexes continue to be of considerable interest on account of their biological properties such as antibacterial (Melník et al., 1982), antifungal (Kozlevčar et al., 1999), cytotoxic and antiviral activities (Ranford et al., 1993). Carboxylate ligands are versatile and can coordinate to metal centers in different modes such as monodentate, bidentate and bridging fashions. The bidentate coordination can be either symmetrical bidentate chelating, having the same $\mathrm{C}-\mathrm{O}$ bond lengths, or asymmetrical bidentate chelating, having different $\mathrm{C}-\mathrm{O}$ bond lengths. Carboxylate ligands have been used to generate units for developing supramolecular architectures. Copper is one of essential metals for human life. In the human body, various enzymes are copper-dependent such as Cytochrome c oxidase, superoxide dismutase, ferroxidases, monoamine oxidase, and dopamine $\beta$-monoxygenase (Brewer, 2009; Balamurugan \& Schaffner, 2006). In this work, a new copper(II) complex involving 2-nitrobenzoic acid and $N, N, N^{\prime}, N^{\prime}$-tetramethylethylenediamine was synthesized, characterized by single crystal X-ray and studied by Hirshfeld surface analysis. 


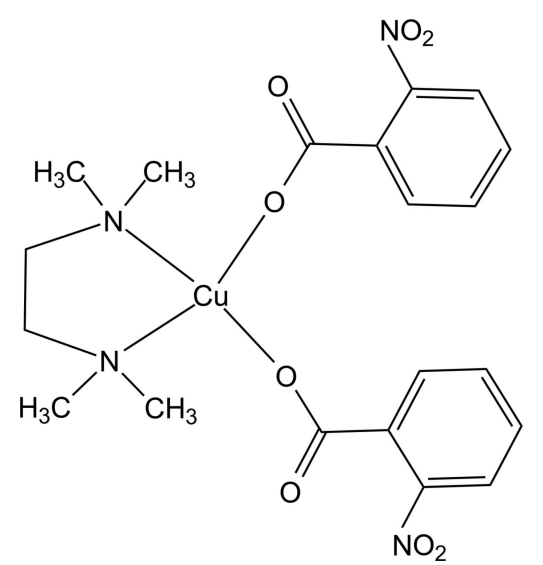

\section{Structural commentary}

Copper(II) acetate reacts with 2-nitrobenzoic acid and $N, N, N^{\prime}, N^{\prime}$-tetramethylethylenediamine (TMEDA) to give the mono-nuclear copper(II) complex (I). The asymmetric unit of the title compound contains one half of the metal complex, the central metal being located on the special position $4 e(1 / 2, y, 1 /$ 4). $\mathrm{The} \mathrm{Cu}^{\mathrm{II}}$ atom has a distorted square-planar geometry with one oxygen atom each from two nitrobenzoic acid ligands and two TMEDA ligand nitrogen atoms (Figs. 1 and 2). The two nitro groups of the rings are oriented trans to each other, being symmetry-related to each other through a twofold axis. The structure of the complex is shown in Fig. 1. The Cu1-N1 and Cu1-O1 bond distances are 2.0269 (13) and 1.9589 (11) $\AA$, respectively. The structural parameters of the TMEDA ligand, i.e. $\mathrm{Cu}-\mathrm{N}$ bond lengths, are in agreement with a work reported by Gumienna-Kontecka et al. (2013). The C4-O1 and $\mathrm{C} 4-\mathrm{O} 2$ distances in the carboxyl group are 1.2772 (19) and 1.2388 (18) $\AA$, respectively. Selected bond lengths are given in Table 1 .

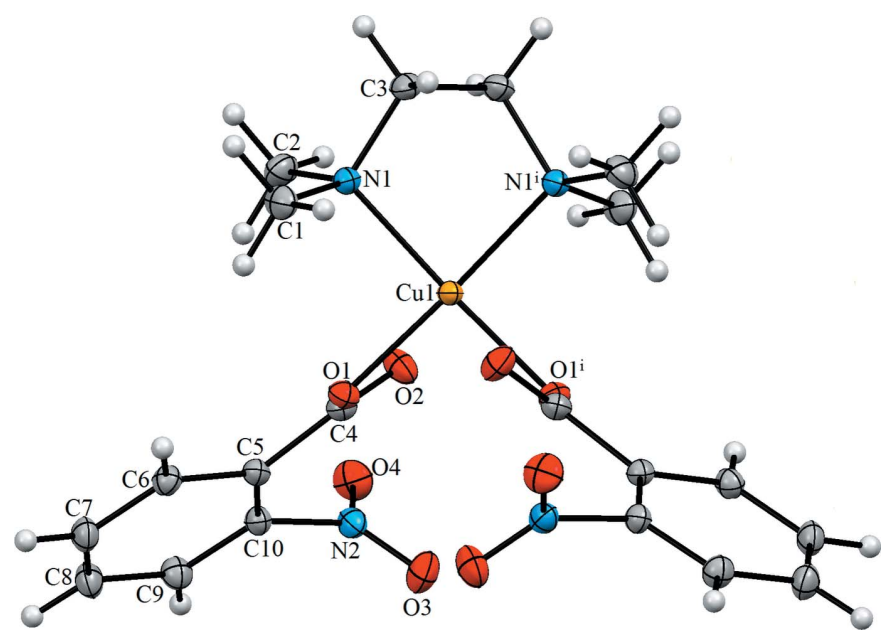

Figure 1

The molecular structure of $\left[\mathrm{Cu}(2 \text {-nitrobenzoate })_{2}\right.$ (tmeda)], with the atom labeling. Displacement ellipsoids are drawn at the $30 \%$ probability level. Symmetry code: (i) $-x+1, y,-z+\frac{1}{2}$.
Table 1

Selected bond lengths $(\AA)$.

\begin{tabular}{llll}
\hline $\mathrm{C} 1-\mathrm{N} 1$ & $1.482(2)$ & $\mathrm{Cu} 1-\mathrm{O} 1$ & $1.9589(11)$ \\
$\mathrm{C} 2-\mathrm{N} 1$ & $1.483(2)$ & $\mathrm{Cu} 1-\mathrm{N} 1$ & $2.0269(13)$ \\
$\mathrm{C} 3-\mathrm{N} 1$ & $1.490(2)$ & $\mathrm{N} 2-\mathrm{O} 4$ & $1.2206(18)$ \\
$\mathrm{C} 10-\mathrm{N} 2$ & $1.4742(19)$ & $\mathrm{N} 2-\mathrm{O} 3$ & $1.2249(19)$ \\
\hline
\end{tabular}

Table 2

Hydrogen-bond geometry $\left(\AA,{ }^{\circ}\right)$.

\begin{tabular}{lllll}
\hline$D-\mathrm{H} \cdots A$ & $D-\mathrm{H}$ & $\mathrm{H} \cdots A$ & $D \cdots A$ & $D-\mathrm{H} \cdots A$ \\
\hline $\mathrm{C} 3-\mathrm{H} 3 A \cdots \mathrm{O} 3^{\mathrm{i}}$ & 0.99 & 2.59 & $3.531(2)$ & 158 \\
$\mathrm{C} 9-\mathrm{H} 9 \cdots \mathrm{O} 4^{\mathrm{ii}}$ & 0.95 & 2.42 & $3.291(2)$ & 152 \\
\hline
\end{tabular}

Symmetry codes: (i) $-x+1, y-1,-z+\frac{1}{2}$; (ii) $-x+\frac{3}{2},-y+\frac{3}{2},-z+1$.

\section{Supramolecular features}

The crystal packing of the title complex (Fig. 2) features intermolecular hydrogen bonds $\left(\mathrm{C} 3-\mathrm{H} 3 A \cdots \mathrm{O}^{\mathrm{i}}\right.$ and $\mathrm{C} 9-$ $\mathrm{H} 9 \cdots \mathrm{O} 4^{\mathrm{ii}}$; symmetry codes as in Table 2). The metal complexes are self-assembled by centrosymmetric $\mathrm{C} 9-$ $\mathrm{H} 9 \cdots \mathrm{O} 4$ hydrogen bonds along the $c$-axis direction, forming supramolecular ribbons linked via $R_{2}^{2}(10)$ ring motifs. Adjacent ribbons are connected by $\mathrm{C} 3-\mathrm{H} 3 A \cdots \mathrm{O} 3$ hydrogen bonds; these interactions lead to the formation of layers lying parallel to the $b c$ plane. The three-dimensional network is stabilized by $\pi-\pi$ stacking interactions with a centroid-tocentroid distance $C g 1 \cdots C g 1^{\text {iii }}$ of 3.741 (2) $\AA$, where $C g 1$ is the centroid of the $\mathrm{C} 5-\mathrm{C} 10$ ring [symmetry code: (iii) $-x+1$, $-y+1,-z+1]$.

\section{Database survey}

A search of the Cambridge Structural Database (CSD, version 5.41, update of November 2019; Groom et al., 2016) for the title complex revealed four hits: catena-[ $\left(\mu_{2}\right.$-terephthalato$\left.O, O^{\prime}, O^{\prime \prime}, O^{\prime \prime \prime}\right)\left(\mu_{2}\right.$-terephthalato- $\left.O, O^{\prime \prime}\right)$ bis $[N$-(2-aminoethyl)3-amino-1-propanol]dicopper(II)] (FEMBEF; Mukherjee et al., 2004), bis $\left[\left(\mu_{2}\right.\right.$-biphenyl-2,2'-dicarboxylato- $\left.O^{2}, O^{2^{\prime}}\right)[N$ (pyrid;in-2-yl- $N$ )pyridin-2-amine- $\left.N^{1}\right]$ ]dicopper(II) tetrahydrate (GUCXOS; Kumagai et al., 2009), bis[( $\mu_{2}$-biphenyl-

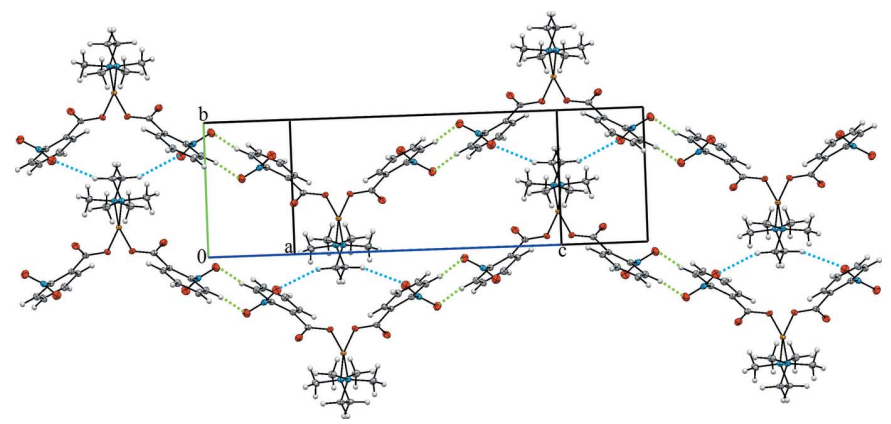

Figure 2

View of the two-dimensional hydrogen-bonded network in the structure of $\left[\mathrm{Cu}(2 \text {-nitrobenzoate })_{2}\right.$ (tmeda) showing $\mathrm{C} 9-\mathrm{H} 9 \cdots \mathrm{O} 4$ hydrogen bonds [described by an $R_{2}^{2}(10)$ ring motif] as green dashed lines and C3$\mathrm{H} 3 A \cdots \mathrm{O} 3$ hydrogen bonds as blue dashed lines. 
2,2'-dicarboxylato- $\left.O^{2}, O^{2^{\prime}}\right)[N$-(pyridin-2-yl- $N$ )pyridin-2-amine- $\left.\left.N^{1}\right]\right]$ dicopper(II) biphenyl-2,2'-dicarboxylic acid solvate monohydrate (GUCXUY; Kumagai et al., 2009) and bis(2nitrobenzoato)bis(3,5-dimethyl-1 $H$-pyrazole- $N^{2}$ ) copper(II) (MIJFUH; Karmakar et al., 2007). The $\mathrm{Cu}-\mathrm{N}$ and $\mathrm{Cu}-\mathrm{O}$ bond lengths range from 1.973 to $2.022 \AA$ and 1.955 to $1.987 \AA$, respectively. The $\mathrm{Cu}-\mathrm{N}$ and $\mathrm{Cu}-\mathrm{O}$ bond lengths in the title complex [2.0269(13) and $1.9589(11) \AA$, respectively] fall within these limits.

\section{Hirshfeld surface analysis}

Hirshfeld surface analysis and the associated two-dimensional fingerprint plots (Spackman \& Jayatilaka, 2009) are very important for explaining the intermolecular contacts in the crystal structure (Demircioğlu et al., 2019; Ilmi et al., 2020). We performed the Hirshfeld surface analysis with CrystalExplorer17 (Turner et al., 2017). Fig. 3 shows the Hirshfeld surface mapped over $d_{\text {norm }}(-0.2250$ to 1.2935 a.u.) and the molecular electrostatic potentials $(-0.2173$ to 0.1248$)$. In Fig. $3 a$, the red spots correspond to the $\mathrm{O} \cdots \mathrm{H}$ contacts. The electrostatic potential (Fig. 3b) shows donor (red) and acceptor (blue) regions. $\mathrm{O} \cdots \mathrm{H} / \mathrm{H} \cdots \mathrm{O}(44.9 \%)$ contacts, seen as a pair of spikes of scattered points in the fingerprint plot, make the largest contribution to the total Hirshfeld surface in $\left[\mathrm{Cu}(2 \text {-nitrobenzoate })_{2}\right.$ (tmeda)] (Fig. 4). The second most important interaction is $\mathrm{H} \cdots \mathrm{H}$, contributing $34 \%$ to the overall crystal packing, which is shown in the 2D fingerprint of the $\left(d_{\mathrm{i}}, d_{\mathrm{e}}\right)$ points related to the $\mathrm{H}$ atoms. Two symmetrical
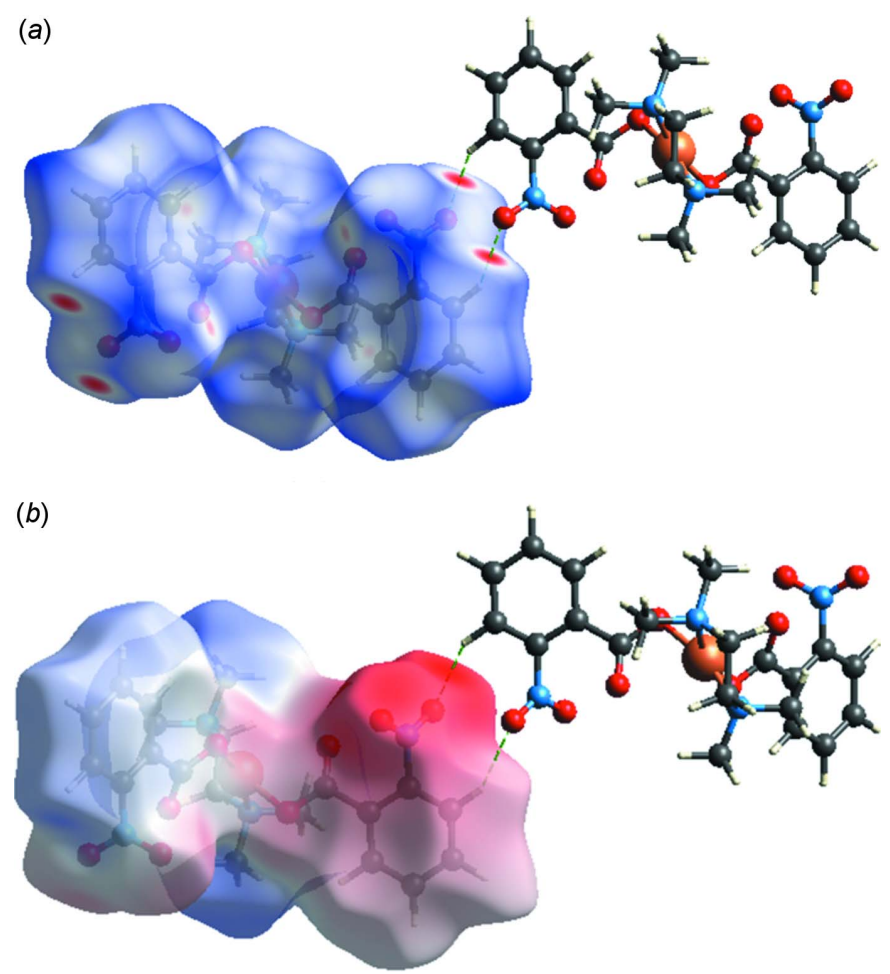

Figure 3

Hirshfeld surface of $\left[\mathrm{Cu}(2 \text {-nitrobenzoate })_{2}\right.$ (tmeda) $]$ mapped with $(a)$ $d_{\text {norm }}$ and $(b)$ the molecular electrostatic potential.
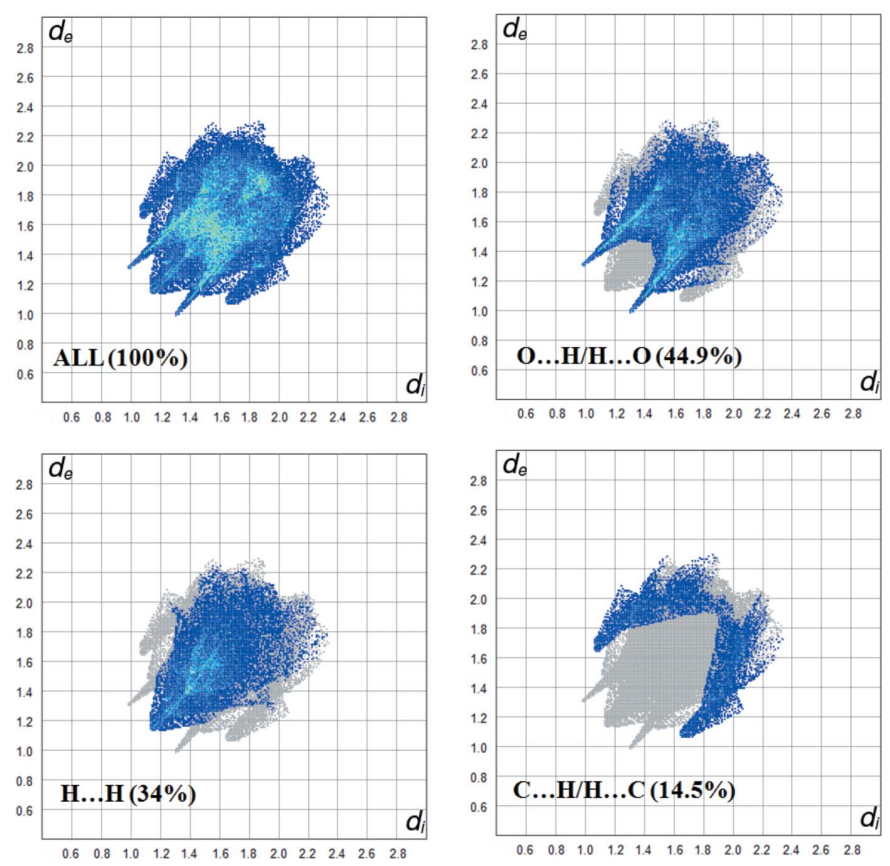

Figure 4

Two-dimensional fingerprint plots for [ $\mathrm{Cu}(2 \text {-nitrobenzoate) })_{2}($ tmeda) $]$ showing all interactions and those delineated into $\mathrm{O} \cdots \mathrm{H} / \mathrm{H} \cdots \mathrm{O}, \mathrm{H} \cdots \mathrm{H}$ and $\mathrm{C} \cdots \mathrm{H} / \mathrm{H} \cdots \mathrm{C}$ contacts $\left(d_{\mathrm{i}}\right.$ is the closest internal distance from a given point on the Hirshfeld surface and $d_{\mathrm{e}}$ is the closest external contact).

wings on the left and right sides are shown in the graph of $\mathrm{C} \cdots \mathrm{H} / \mathrm{H} \cdots \mathrm{C}$ interactions (14.5\%). The Hirshfeld surface analysis confirms the importance of $\mathrm{H}$-atom contacts in establishing the packing. The large number of $\mathrm{O} \cdots \mathrm{H}, \mathrm{H} \cdots \mathrm{H}$ and $\mathrm{C} \cdots \mathrm{H}$ interactions suggest that van der Waals interactions and hydrogen bonding play the major role in the crystal packing.

\section{Synthesis and crystallization}

An aqueous solution of sodium 2-nitrobenzoate $(5 \mathrm{mmol}$, $0.9 \mathrm{~g}$ ) was added to an aqueous solution of $\mathrm{CuSO}_{4} \cdot 5 \mathrm{H}_{2} \mathrm{O}$ $(2.5 \mathrm{mmol}, 0.6 \mathrm{~g})$ under stirring. Tetramethylethylenediamine $(2.5 \mathrm{mmol}, 0.3 \mathrm{~g}$ ) was added and the color changed from light blue to violet. The mixture was filtered and the filtrate was allowed to stand for slow evaporation. Single crystals suitable for X-ray were obtained after several days.

\section{Refinement}

Crystal data, data collection and structure refinement details are summarized in Table 3. C-bound $\mathrm{H}$ atoms were positioned geometrically $(\mathrm{C}-\mathrm{H}=0.95,0.98$ and $0.99 \AA)$ and refined using a riding model, with $U_{\text {iso }}(\mathrm{H})=1.5 U_{\text {eq }}(\mathrm{C})$ for methyl $\mathrm{H}$ atoms and $1.2 U_{\text {eq }}(\mathrm{C})$ otherwise.

\section{Acknowledgements}

Authors contributions are as follows. Methodology, AMQ; software, SK, ND, LY and ES; validation, SK, AMQ and ND; formal analysis, AMQ; investigation, SK, AMQ, ND and ES; 
Table 3

Experimental details.

\begin{tabular}{|c|c|}
\hline \multicolumn{2}{|l|}{ Crystal data } \\
\hline Chemical formula & {$\left[\mathrm{Cu}\left(\mathrm{C}_{7} \mathrm{H}_{4} \mathrm{NO}_{4}\right)_{2}\left(\mathrm{C}_{6} \mathrm{H}_{16} \mathrm{~N}_{2}\right)\right]$} \\
\hline$M_{\mathrm{r}}$ & 511.97 \\
\hline Crystal system, space group & Monoclinic, $C 2 / c$ \\
\hline Temperature $(\mathrm{K})$ & 100 \\
\hline$a, b, c(\AA)$ & $12.7286(3), 7.4918(2), 22.8967(6)$ \\
\hline$\beta\left(^{\circ}\right)$ & $98.395(1)$ \\
\hline$V\left(\AA^{3}\right)$ & $2160.04(10)$ \\
\hline$Z$ & 4 \\
\hline Radiation type & Mo $K \alpha$ \\
\hline$\mu\left(\mathrm{mm}^{-1}\right)$ & 1.07 \\
\hline Crystal size $(\mathrm{mm})$ & $0.22 \times 0.20 \times 0.12$ \\
\hline \multicolumn{2}{|l|}{ Data collection } \\
\hline Diffractometer & $\begin{array}{l}\text { Bruker D8 Quest withPhoton II } \\
\text { CPADs detector }\end{array}$ \\
\hline Absorption correction & $\begin{array}{l}\text { Multi-scan (SADABS; Bruker, } \\
\text { 2017) }\end{array}$ \\
\hline$T_{\min }, T_{\max }$ & $0.77,0.88$ \\
\hline $\begin{array}{l}\text { No. of measured, independent and } \\
\text { observed }[I>2 \sigma(I)] \text { reflections }\end{array}$ & $23494,4737,3573$ \\
\hline$R_{\text {int }}$ & 0.056 \\
\hline$(\sin \theta / \lambda)_{\max }\left(\AA^{-1}\right)$ & 0.808 \\
\hline \multicolumn{2}{|l|}{ Refinement } \\
\hline$R\left[F^{2}>2 \sigma\left(F^{2}\right)\right], w R\left(F^{2}\right), S$ & $0.042,0.092,1.03$ \\
\hline No. of reflections & 4737 \\
\hline No. of parameters & 152 \\
\hline H-atom treatment & H-atom parameters constrained \\
\hline$\Delta \rho_{\max }, \Delta \rho_{\min }\left(\mathrm{e} \AA^{-3}\right)$ & $0.55,-0.63$ \\
\hline
\end{tabular}

Computer programs: APEX3 and SAINT (Bruker, 2017), SHELXT2014/5 (Sheldrick, 2015a) and SHELXL2017/1 (Sheldrick, 2015b).

resources, AMQ and ES; writing (review and editing), SK and AMQ; visualization, SK; supervision, SK and ND; funding acquisition, LY and ES.

\section{References}

Balamurugan, K. \& Schaffner, W. (2006). BBA Mol. Cell. Res. 1763, 737-746.

Brewer, G. J. (2009). J. Am. Coll. Nutr. 28, 238-242.

Bruker (2017). APEX3, SADABS and SAINT. Bruker AXS Inc., Madison, Wisconsin, USA.

Demircioğlu, Z., Kaştaş, G., Kaştaş, Ç. A. \& Frank, R. (2019). J. Mol. Struct. 1191, 129-137.

Groom, C. R., Bruno, I. J., Lightfoot, M. P. \& Ward, S. C. (2016). Acta Cryst. B72, 171-179.

Gumienna-Kontecka, E., Golenya, I. A., Szebesczyk, A., Haukka, M., Krämer, R. \& Fritsky, I. O. (2013). Inorg. Chem. 52, 7633 7644.

Ilmi, R., Kansız, S., Al-Rasbi, N. K., Dege, N., Raithby, P. R. \& Khan, M. S. (2020). New J. Chem. 44, 5673-5683.

Karmakar, A., Bania, K., Baruah, A. M. \& Baruah, J. B. (2007). Inorg. Chem. Commun. 10, 959-964.

Kozlevčar, B., Leban, I., Turel, I., Šegedin, P., Petric, M., Pohleven, F., White, A., Williams, D. \& Sieler, J. (1999). Polyhedron, 18, 755762.

Kumagai, H., Akita-Tanaka, M., Kawata, S., Inoue, K., Kepert, C. J. \& Kurmoo, M. (2009). Cryst. Growth Des. 9, 2734-2741.

Melník, M., Auderová, M. \& Hol'ko, M. (1982). Inorg. Chim. Acta, 67, 117-120.

Mukherjee, P. S., Ghoshal, D., Zangrando, E., Mallah, T. \& Chaudhuri, N. R. (2004). Eur. J. Inorg. Chem. pp. 4675-4680.

Ranford, J. D., Sadler, P. J. \& Tocher, D. A. (1993). J. Chem. Soc. Dalton Trans. pp. 3393-3399.

Sheldrick, G. M. (2015a). Acta Cryst. A71, 3-8.

Sheldrick, G. M. (2015b). Acta Cryst. C71, 3-8.

Spackman, M. A. \& Jayatilaka, D. (2009). CrystEngComm, 11, 1932.

Turner, M. J., MacKinnon, J. J., Wolff, S. K., Grimwood, D. J., Spackman, P. R., Jayatilaka, D. \& Spackman, M. A. (2017). CrystalExplorer17.5. University of Western Australia. http:// hirshfeldsurface.net. 


\section{supporting information}

Acta Cryst. (2021). E77, 412-415 [https://doi.org/10.1107/S2056989021002802]

\section{Crystal structure and Hirshfeld surface analysis of a copper(II) complex}

\section{containing 2-nitrobenzoate and tetramethylethylenediamine ligands}

\section{Sevgi Kansiz, Adnan M. Qadir, Necmi Dege, Li Yongxin and Eiad Saif}

Computing details

Data collection: APEX3 (Bruker, 2017); cell refinement: SAINT (Bruker, 2017); data reduction: SAINT (Bruker, 2017); program(s) used to solve structure: SHELXT2014/5 (Sheldrick, 2015a); program(s) used to refine structure: SHELXL2017/1 (Sheldrick, 2015b); software used to prepare material for publication: APEX3 (Bruker, 2017).

Bis(2-nitrobenzoato- $\kappa O)\left(N, N_{,} N^{\prime}, N^{\prime}\right.$-tetramethylethylenediamine- $\left.\kappa^{2} N, N^{\prime}\right) \operatorname{copper}($ II)

Crystal data

$\left[\mathrm{Cu}\left(\mathrm{C}_{7} \mathrm{H}_{4} \mathrm{NO}_{4}\right)_{2}\left(\mathrm{C}_{6} \mathrm{H}_{16} \mathrm{~N}_{2}\right)\right]$

$M_{r}=511.97$

Monoclinic, $C 2 / \mathrm{c}$

$a=12.7286(3) \AA$

$b=7.4918(2) \AA$

$c=22.8967(6) \AA$

$\beta=98.395(1)^{\circ}$

$V=2160.04(10) \AA^{3}$

$Z=4$

Data collection

Bruker D8 Quest withPhoton II CPADs detector diffractometer

Radiation source: Incoatec microfocus source, Bruker D8 Quest

Multilayer Mirror monochromator

Detector resolution: 7.4074 pixels $\mathrm{mm}^{-1}$

phi and $\omega$ scans

Absorption correction: multi-scan

(SADABS; Bruker, 2017)

\section{Refinement}

Refinement on $F^{2}$

Least-squares matrix: full

$R\left[F^{2}>2 \sigma\left(F^{2}\right)\right]=0.042$

$w R\left(F^{2}\right)=0.092$

$S=1.03$

4737 reflections

152 parameters

0 restraints

Primary atom site location: dual
$F(000)=1060$

$D_{\mathrm{x}}=1.574 \mathrm{Mg} \mathrm{m}^{-3}$

Mo $K \alpha$ radiation, $\lambda=0.71073 \AA$

Cell parameters from 6607 reflections

$\theta=3.2-34.7^{\circ}$

$\mu=1.07 \mathrm{~mm}^{-1}$

$T=100 \mathrm{~K}$

Block, blue

$0.22 \times 0.20 \times 0.12 \mathrm{~mm}$

$T_{\min }=0.77, T_{\max }=0.88$

23494 measured reflections

4737 independent reflections

3573 reflections with $I>2 \sigma(I)$

$R_{\text {int }}=0.056$

$\theta_{\max }=35.1^{\circ}, \theta_{\min }=3.2^{\circ}$

$h=-20 \rightarrow 20$

$k=-12 \rightarrow 12$

$l=-36 \rightarrow 34$

Hydrogen site location: inferred from neighbouring sites

$\mathrm{H}$-atom parameters constrained

$w=1 /\left[\sigma^{2}\left(F_{\mathrm{o}}{ }^{2}\right)+(0.0258 P)^{2}+3.2488 P\right]$

where $P=\left(F_{\mathrm{o}}^{2}+2 F_{\mathrm{c}}^{2}\right) / 3$

$(\Delta / \sigma)_{\max }<0.001$

$\Delta \rho_{\max }=0.55{\mathrm{e} \AA^{-3}}^{-3}$

$\Delta \rho_{\text {min }}=-0.63$ e $\AA^{-3}$ 


\section{Special details}

Geometry. All esds (except the esd in the dihedral angle between two 1.s. planes) are estimated using the full covariance matrix. The cell esds are taken into account individually in the estimation of esds in distances, angles and torsion angles; correlations between esds in cell parameters are only used when they are defined by crystal symmetry. An approximate (isotropic) treatment of cell esds is used for estimating esds involving l.s. planes.

Fractional atomic coordinates and isotropic or equivalent isotropic displacement parameters $\left(\AA^{2}\right)$

\begin{tabular}{|c|c|c|c|c|}
\hline & $x$ & $y$ & $z$ & $U_{\text {iso }} * / U_{\text {eq }}$ \\
\hline $\mathrm{C} 1$ & $0.29927(12)$ & $0.0971(2)$ & $0.26549(9)$ & $0.0226(3)$ \\
\hline H1A & 0.282285 & 0.110738 & 0.222582 & $0.034^{*}$ \\
\hline H1B & 0.282610 & 0.208122 & 0.284815 & $0.034^{*}$ \\
\hline $\mathrm{H} 1 \mathrm{C}$ & 0.257167 & -0.000768 & 0.278536 & $0.034^{*}$ \\
\hline $\mathrm{C} 2$ & $0.43747(14)$ & $0.0376(2)$ & $0.34649(8)$ & $0.0227(3)$ \\
\hline $\mathrm{H} 2 \mathrm{~A}$ & 0.395284 & -0.060381 & 0.359361 & $0.034^{*}$ \\
\hline $\mathrm{H} 2 \mathrm{~B}$ & 0.419573 & 0.148951 & 0.365245 & $0.034^{*}$ \\
\hline $\mathrm{H} 2 \mathrm{C}$ & 0.513178 & 0.011891 & 0.357879 & $0.034^{*}$ \\
\hline $\mathrm{C} 3$ & $0.44109(12)$ & $-0.1106(2)$ & $0.25164(8)$ & $0.0191(3)$ \\
\hline $\mathrm{H} 3 \mathrm{~A}$ & 0.400440 & -0.116874 & 0.211384 & $0.023^{*}$ \\
\hline H3B & 0.422415 & -0.215879 & 0.274155 & $0.023^{*}$ \\
\hline $\mathrm{C} 4$ & $0.51601(12)$ & $0.4529(2)$ & $0.34319(7)$ & $0.0161(3)$ \\
\hline $\mathrm{C} 5$ & $0.48616(11)$ & $0.56860(19)$ & $0.39205(7)$ & $0.0141(3)$ \\
\hline C6 & $0.37979(12)$ & $0.5852(2)$ & $0.39976(7)$ & $0.0164(3)$ \\
\hline H6 & 0.326473 & 0.529299 & 0.372478 & $0.020^{*}$ \\
\hline $\mathrm{C} 7$ & $0.35056(12)$ & $0.6815(2)$ & $0.44637(8)$ & $0.0196(3)$ \\
\hline H7 & 0.277599 & 0.693902 & 0.450177 & $0.024^{*}$ \\
\hline $\mathrm{C} 8$ & $0.42749(12)$ & $0.7599(2)$ & $0.48755(7)$ & $0.0205(3)$ \\
\hline H8 & 0.407256 & 0.823813 & 0.519973 & $0.025^{*}$ \\
\hline C9 & $0.53414(12)$ & $0.7451(2)$ & $0.48142(7)$ & $0.0188(3)$ \\
\hline H9 & 0.587555 & 0.797819 & 0.509431 & $0.023^{*}$ \\
\hline $\mathrm{C} 10$ & 0.56064 (11) & $0.6518(2)$ & $0.43364(7)$ & $0.0151(3)$ \\
\hline $\mathrm{Cu} 1$ & 0.500000 & $0.25244(3)$ & 0.250000 & $0.01202(6)$ \\
\hline N1 & $0.41383(10)$ & $0.05640(17)$ & $0.28142(6)$ & $0.0152(2)$ \\
\hline N2 & $0.67360(10)$ & $0.65314(18)$ & $0.42590(6)$ & $0.0176(3)$ \\
\hline $\mathrm{O} 1$ & $0.44317(9)$ & $0.43232(14)$ & $0.29904(5)$ & $0.0165(2)$ \\
\hline $\mathrm{O} 2$ & $0.60401(9)$ & $0.37907(16)$ & $0.34882(6)$ & $0.0226(2)$ \\
\hline $\mathrm{O} 3$ & $0.69761(10)$ & $0.73929(18)$ & $0.38435(6)$ & $0.0275(3)$ \\
\hline $\mathrm{O} 4$ & $0.73708(9)$ & $0.57614(18)$ & $0.46249(6)$ & $0.0268(3)$ \\
\hline
\end{tabular}

Atomic displacement parameters $\left(\AA^{2}\right)$

\begin{tabular}{lllllll}
\hline & $U^{11}$ & $U^{22}$ & $U^{33}$ & $U^{12}$ & $U^{13}$ & $U^{23}$ \\
\hline C1 & $0.0144(6)$ & $0.0213(7)$ & $0.0324(10)$ & $-0.0007(6)$ & $0.0050(6)$ & $-0.0020(7)$ \\
C2 & $0.0297(8)$ & $0.0196(7)$ & $0.0197(8)$ & $-0.0011(6)$ & $0.0063(7)$ & $0.0039(6)$ \\
C3 & $0.0187(6)$ & $0.0126(6)$ & $0.0265(8)$ & $-0.0024(5)$ & $0.0054(6)$ & $-0.0010(6)$ \\
C4 & $0.0175(6)$ & $0.0128(6)$ & $0.0184(7)$ & $-0.0012(5)$ & $0.0039(5)$ & $-0.0002(5)$ \\
C5 & $0.0144(6)$ & $0.0124(6)$ & $0.0159(7)$ & $0.0005(5)$ & $0.0030(5)$ & $0.0005(5)$ \\
C6 & $0.0140(6)$ & $0.0167(6)$ & $0.0187(7)$ & $-0.0013(5)$ & $0.0025(5)$ & $0.0003(6)$
\end{tabular}


supporting information

\begin{tabular}{lllllll} 
C7 & $0.0161(6)$ & $0.0231(7)$ & $0.0211(8)$ & $0.0007(6)$ & $0.0073(6)$ & $0.0002(6)$ \\
C8 & $0.0201(6)$ & $0.0241(7)$ & $0.0189(7)$ & $0.0001(6)$ & $0.0073(6)$ & $-0.0038(7)$ \\
C9 & $0.0178(6)$ & $0.0204(6)$ & $0.0181(7)$ & $-0.0009(6)$ & $0.0026(5)$ & $-0.0021(6)$ \\
C10 & $0.0131(6)$ & $0.0149(6)$ & $0.0177(7)$ & $0.0004(5)$ & $0.0036(5)$ & $-0.0002(5)$ \\
Cu1 & $0.01247(10)$ & $0.01009(10)$ & $0.01356(12)$ & 0.000 & $0.00210(8)$ & 0.000 \\
N1 & $0.0149(5)$ & $0.0127(5)$ & $0.0186(6)$ & $-0.0002(4)$ & $0.0040(5)$ & $0.0006(5)$ \\
N2 & $0.0145(5)$ & $0.0174(6)$ & $0.0214(7)$ & $-0.0017(5)$ & $0.0037(5)$ & $-0.0035(5)$ \\
O1 & $0.0189(5)$ & $0.0143(5)$ & $0.0161(5)$ & $0.0004(4)$ & $0.0021(4)$ & $-0.0023(4)$ \\
O2 & $0.0180(5)$ & $0.0237(6)$ & $0.0262(6)$ & $0.0038(4)$ & $0.0038(5)$ & $-0.0065(5)$ \\
O3 & $0.0213(5)$ & $0.0332(7)$ & $0.0297(7)$ & $-0.0039(5)$ & $0.0092(5)$ & $0.0044(6)$ \\
O4 & $0.0165(5)$ & $0.0295(7)$ & $0.0329(7)$ & $0.0040(5)$ & $-0.0013(5)$ & $0.0026(6)$ \\
\hline
\end{tabular}

Geometric parameters $\left(\AA,{ }^{\circ}\right)$

\begin{tabular}{|c|c|c|c|}
\hline $\mathrm{C} 1-\mathrm{N} 1$ & $1.482(2)$ & $\mathrm{C} 5-\mathrm{C} 6$ & $1.396(2)$ \\
\hline $\mathrm{C} 1-\mathrm{H} 1 \mathrm{~A}$ & 0.9800 & $\mathrm{C} 6-\mathrm{C} 7$ & $1.383(2)$ \\
\hline $\mathrm{C} 1-\mathrm{H} 1 \mathrm{~B}$ & 0.9800 & $\mathrm{C} 6-\mathrm{H} 6$ & 0.9500 \\
\hline $\mathrm{C} 1-\mathrm{H} 1 \mathrm{C}$ & 0.9800 & $\mathrm{C} 7-\mathrm{C} 8$ & $1.387(2)$ \\
\hline $\mathrm{C} 2-\mathrm{N} 1$ & $1.483(2)$ & $\mathrm{C} 7-\mathrm{H} 7$ & 0.9500 \\
\hline $\mathrm{C} 2-\mathrm{H} 2 \mathrm{~A}$ & 0.9800 & $\mathrm{C} 8-\mathrm{C} 9$ & $1.389(2)$ \\
\hline $\mathrm{C} 2-\mathrm{H} 2 \mathrm{~B}$ & 0.9800 & $\mathrm{C} 8-\mathrm{H} 8$ & 0.9500 \\
\hline $\mathrm{C} 2-\mathrm{H} 2 \mathrm{C}$ & 0.9800 & $\mathrm{C} 9-\mathrm{C} 10$ & $1.381(2)$ \\
\hline $\mathrm{C} 3-\mathrm{N} 1$ & $1.490(2)$ & $\mathrm{C} 9-\mathrm{H} 9$ & 0.9500 \\
\hline $\mathrm{C} 3-\mathrm{C}^{\mathrm{i}}$ & $1.513(3)$ & $\mathrm{C} 10-\mathrm{N} 2$ & $1.4742(19)$ \\
\hline $\mathrm{C} 3-\mathrm{H} 3 \mathrm{~A}$ & 0.9900 & $\mathrm{Cu} 1-\mathrm{O} 1$ & $1.9589(11)$ \\
\hline $\mathrm{C} 3-\mathrm{H} 3 \mathrm{~B}$ & 0.9900 & $\mathrm{Cu} 1-\mathrm{O} 1^{\mathrm{i}}$ & $1.9589(11)$ \\
\hline $\mathrm{C} 4-\mathrm{O} 2$ & $1.2388(18)$ & $\mathrm{Cu} 1-\mathrm{N} 1$ & $2.0269(13)$ \\
\hline $\mathrm{C} 4-\mathrm{O} 1$ & $1.2772(19)$ & $\mathrm{Cu} 1-\mathrm{N} 1^{\mathrm{i}}$ & $2.0269(13)$ \\
\hline $\mathrm{C} 4-\mathrm{C} 5$ & $1.508(2)$ & $\mathrm{N} 2-\mathrm{O} 4$ & $1.2206(18)$ \\
\hline $\mathrm{C} 5-\mathrm{C} 10$ & $1.389(2)$ & $\mathrm{N} 2-\mathrm{O} 3$ & 1.2249 (19) \\
\hline $\mathrm{N} 1-\mathrm{C} 1-\mathrm{H} 1 \mathrm{~A}$ & 109.5 & $\mathrm{C} 6-\mathrm{C} 7-\mathrm{H} 7$ & 119.9 \\
\hline $\mathrm{N} 1-\mathrm{C} 1-\mathrm{H} 1 \mathrm{~B}$ & 109.5 & $\mathrm{C} 8-\mathrm{C} 7-\mathrm{H} 7$ & 119.9 \\
\hline $\mathrm{H} 1 \mathrm{~A}-\mathrm{C} 1-\mathrm{H} 1 \mathrm{~B}$ & 109.5 & $\mathrm{C} 7-\mathrm{C} 8-\mathrm{C} 9$ & $120.04(15)$ \\
\hline $\mathrm{N} 1-\mathrm{C} 1-\mathrm{H} 1 \mathrm{C}$ & 109.5 & $\mathrm{C} 7-\mathrm{C} 8-\mathrm{H} 8$ & 120.0 \\
\hline $\mathrm{H} 1 \mathrm{~A}-\mathrm{C} 1-\mathrm{H} 1 \mathrm{C}$ & 109.5 & $\mathrm{C} 9-\mathrm{C} 8-\mathrm{H} 8$ & 120.0 \\
\hline $\mathrm{H} 1 \mathrm{~B}-\mathrm{C} 1-\mathrm{H} 1 \mathrm{C}$ & 109.5 & $\mathrm{C} 10-\mathrm{C} 9-\mathrm{C} 8$ & $118.42(14)$ \\
\hline $\mathrm{N} 1-\mathrm{C} 2-\mathrm{H} 2 \mathrm{~A}$ & 109.5 & $\mathrm{C} 10-\mathrm{C} 9-\mathrm{H} 9$ & 120.8 \\
\hline $\mathrm{N} 1-\mathrm{C} 2-\mathrm{H} 2 \mathrm{~B}$ & 109.5 & $\mathrm{C} 8-\mathrm{C} 9-\mathrm{H} 9$ & 120.8 \\
\hline $\mathrm{H} 2 \mathrm{~A}-\mathrm{C} 2-\mathrm{H} 2 \mathrm{~B}$ & 109.5 & $\mathrm{C} 9-\mathrm{C} 10-\mathrm{C} 5$ & $123.28(14)$ \\
\hline $\mathrm{N} 1-\mathrm{C} 2-\mathrm{H} 2 \mathrm{C}$ & 109.5 & $\mathrm{C} 9-\mathrm{C} 10-\mathrm{N} 2$ & $116.55(13)$ \\
\hline $\mathrm{H} 2 \mathrm{~A}-\mathrm{C} 2-\mathrm{H} 2 \mathrm{C}$ & 109.5 & $\mathrm{C} 5-\mathrm{C} 10-\mathrm{N} 2$ & $120.05(13)$ \\
\hline $\mathrm{H} 2 \mathrm{~B}-\mathrm{C} 2-\mathrm{H} 2 \mathrm{C}$ & 109.5 & $\mathrm{O} 1-\mathrm{Cu} 1-\mathrm{O} 1^{\mathrm{i}}$ & 93.07 (7) \\
\hline $\mathrm{N} 1-\mathrm{C} 3-\mathrm{C} 3^{\mathrm{i}}$ & $108.76(11)$ & $\mathrm{O} 1-\mathrm{Cu} 1-\mathrm{N} 1$ & $91.76(5)$ \\
\hline $\mathrm{N} 1-\mathrm{C} 3-\mathrm{H} 3 \mathrm{~A}$ & 109.9 & $\mathrm{O} 1-\mathrm{Cu} 1-\mathrm{N} 1$ & $165.06(5)$ \\
\hline $\mathrm{C} 33^{\mathrm{i}}-\mathrm{C} 3-\mathrm{H} 3 \mathrm{~A}$ & 109.9 & $\mathrm{O} 1-\mathrm{Cu} 1-\mathrm{N} 1^{\mathrm{i}}$ & $165.06(5)$ \\
\hline $\mathrm{N} 1-\mathrm{C} 3-\mathrm{H} 3 \mathrm{~B}$ & 109.9 & $\mathrm{O} 1^{\mathrm{i}}-\mathrm{Cu} 1-\mathrm{N} 1^{\mathrm{i}}$ & $91.76(5)$ \\
\hline $\mathrm{C} 33^{\mathrm{i}}-\mathrm{C} 3-\mathrm{H} 3 \mathrm{~B}$ & 109.9 & $\mathrm{~N} 1-\mathrm{Cu} 1-\mathrm{N} 1^{\mathrm{i}}$ & $87.13(7)$ \\
\hline
\end{tabular}




\begin{tabular}{|c|c|c|c|}
\hline $\mathrm{H} 3 \mathrm{~A}-\mathrm{C} 3-\mathrm{H} 3 \mathrm{~B}$ & 108.3 & $\mathrm{C} 1-\mathrm{N} 1-\mathrm{C} 2$ & $108.35(13)$ \\
\hline $\mathrm{O} 2-\mathrm{C} 4-\mathrm{O} 1$ & $124.71(15)$ & $\mathrm{C} 1-\mathrm{N} 1-\mathrm{C} 3$ & $110.27(12)$ \\
\hline $\mathrm{O} 2-\mathrm{C} 4-\mathrm{C} 5$ & $120.10(14)$ & $\mathrm{C} 2-\mathrm{N} 1-\mathrm{C} 3$ & $110.70(13)$ \\
\hline $\mathrm{O} 1-\mathrm{C} 4-\mathrm{C} 5$ & $115.09(13)$ & $\mathrm{C} 1-\mathrm{N} 1-\mathrm{Cu} 1$ & $109.12(10)$ \\
\hline $\mathrm{C} 10-\mathrm{C} 5-\mathrm{C} 6$ & $116.79(14)$ & $\mathrm{C} 2-\mathrm{N} 1-\mathrm{Cu} 1$ & $112.61(10)$ \\
\hline $\mathrm{C} 10-\mathrm{C} 5-\mathrm{C} 4$ & $123.09(13)$ & $\mathrm{C} 3-\mathrm{N} 1-\mathrm{Cu} 1$ & $105.77(9)$ \\
\hline $\mathrm{C} 6-\mathrm{C} 5-\mathrm{C} 4$ & $119.95(13)$ & $\mathrm{O} 4-\mathrm{N} 2-\mathrm{O} 3$ & $124.54(14)$ \\
\hline $\mathrm{C} 7-\mathrm{C} 6-\mathrm{C} 5$ & $121.26(14)$ & $\mathrm{O} 4-\mathrm{N} 2-\mathrm{C} 10$ & $118.28(14)$ \\
\hline $\mathrm{C} 7-\mathrm{C} 6-\mathrm{H} 6$ & 119.4 & $\mathrm{O} 3-\mathrm{N} 2-\mathrm{C} 10$ & $117.09(13)$ \\
\hline $\mathrm{C} 5-\mathrm{C} 6-\mathrm{H} 6$ & 119.4 & $\mathrm{C} 4-\mathrm{O} 1-\mathrm{Cu} 1$ & $104.50(9)$ \\
\hline $\mathrm{C} 6-\mathrm{C} 7-\mathrm{C} 8$ & $120.18(14)$ & & \\
\hline $\mathrm{O} 2-\mathrm{C} 4-\mathrm{C} 5-\mathrm{C} 10$ & $24.2(2)$ & $\mathrm{C} 4-\mathrm{C} 5-\mathrm{C} 10-\mathrm{C} 9$ & $-174.19(15)$ \\
\hline $\mathrm{O} 1-\mathrm{C} 4-\mathrm{C} 5-\mathrm{C} 10$ & $-159.09(14)$ & $\mathrm{C} 6-\mathrm{C} 5-\mathrm{C} 10-\mathrm{N} 2$ & $-174.93(13)$ \\
\hline $\mathrm{O} 2-\mathrm{C} 4-\mathrm{C} 5-\mathrm{C} 6$ & $-150.89(15)$ & $\mathrm{C} 4-\mathrm{C} 5-\mathrm{C} 10-\mathrm{N} 2$ & $9.9(2)$ \\
\hline $\mathrm{O} 1-\mathrm{C} 4-\mathrm{C} 5-\mathrm{C} 6$ & $25.8(2)$ & $\mathrm{C} 33^{\mathrm{i}}-\mathrm{C} 3-\mathrm{N} 1-\mathrm{C} 1$ & $157.97(16)$ \\
\hline $\mathrm{C} 10-\mathrm{C} 5-\mathrm{C} 6-\mathrm{C} 7$ & $0.6(2)$ & $\mathrm{C} 3{ }^{\mathrm{i}}-\mathrm{C} 3-\mathrm{N} 1-\mathrm{C} 2$ & $-82.14(18)$ \\
\hline $\mathrm{C} 4-\mathrm{C} 5-\mathrm{C} 6-\mathrm{C} 7$ & $175.98(15)$ & $\mathrm{C} 3-\mathrm{C} 3-\mathrm{N} 1-\mathrm{Cu} 1$ & $40.11(18)$ \\
\hline $\mathrm{C} 5-\mathrm{C} 6-\mathrm{C} 7-\mathrm{C} 8$ & $-1.8(2)$ & $\mathrm{C} 9-\mathrm{C} 10-\mathrm{N} 2-\mathrm{O} 4$ & $67.41(19)$ \\
\hline $\mathrm{C} 6-\mathrm{C} 7-\mathrm{C} 8-\mathrm{C} 9$ & $1.3(3)$ & $\mathrm{C} 5-\mathrm{C} 10-\mathrm{N} 2-\mathrm{O} 4$ & $-116.36(17)$ \\
\hline $\mathrm{C} 7-\mathrm{C} 8-\mathrm{C} 9-\mathrm{C} 10$ & $0.2(2)$ & $\mathrm{C} 9-\mathrm{C} 10-\mathrm{N} 2-\mathrm{O} 3$ & $-109.26(17)$ \\
\hline $\mathrm{C} 8-\mathrm{C} 9-\mathrm{C} 10-\mathrm{C} 5$ & $-1.5(2)$ & $\mathrm{C} 5-\mathrm{C} 10-\mathrm{N} 2-\mathrm{O} 3$ & $66.97(19)$ \\
\hline $\mathrm{C} 8-\mathrm{C} 9-\mathrm{C} 10-\mathrm{N} 2$ & $174.64(15)$ & $\mathrm{O} 2-\mathrm{C} 4-\mathrm{O} 1-\mathrm{Cu} 1$ & 4.07 (19) \\
\hline $\mathrm{C} 6-\mathrm{C} 5-\mathrm{C} 10-\mathrm{C} 9$ & $1.0(2)$ & $\mathrm{C} 5-\mathrm{C} 4-\mathrm{O} 1-\mathrm{Cu} 1$ & $-172.47(10)$ \\
\hline
\end{tabular}

Symmetry code: (i) $-x+1, y,-z+1 / 2$.

Hydrogen-bond geometry $\left(A,{ }^{\circ}\right)$

\begin{tabular}{lllll}
\hline$D-\mathrm{H} \cdots A$ & $D-\mathrm{H}$ & $\mathrm{H} \cdots A$ & $D \cdots A$ & $D-\mathrm{H} \cdots A$ \\
\hline $\mathrm{C} 3-\mathrm{H} 3 A \cdots \mathrm{O} 3^{\mathrm{ii}}$ & 0.99 & 2.59 & $3.531(2)$ & 158 \\
$\mathrm{C} 9-\mathrm{H} 9 \cdots \mathrm{O} 44^{\mathrm{iii}}$ & 0.95 & 2.42 & $3.291(2)$ & 152 \\
\hline
\end{tabular}

Symmetry codes: (ii) $-x+1, y-1,-z+1 / 2$; (iii) $-x+3 / 2,-y+3 / 2,-z+1$. 